\title{
Radioiodine Accumulation in a Giant Ovarian Cystadenofibroma Detected Incidentally by 131-I Whole Body Scans
}

\author{
Mohammed Mebarki, ${ }^{1,2}$ Abdelghani Menemani, ${ }^{2}$ \\ Abdelkader Medjahedi, ${ }^{1,2}$ Fouad Boualou, ${ }^{1,3}$ Abdelhak Slama, ${ }^{1,2}$ Sarah Ouguirti, ${ }^{2}$ \\ Fatima Zahra Kherbouche, ${ }^{1,2}$ and Nécib Berber ${ }^{1,2}$ \\ ${ }^{1}$ Faculty of Medicine, University of Tlemcen, Tlemcen 13000, Algeria \\ ${ }^{2}$ Department of Nuclear Medicine, University Hospital of Tlemcen, Tlemcen 13000, Algeria \\ ${ }^{3}$ Department of Surgery “A”, University Hospital of Tlemcen, Tlemcen 13000, Algeria
}

Correspondence should be addressed to Mohammed Mebarki, mmebarki2000@yahoo.fr

Received 12 September 2012; Accepted 1 October 2012

Academic Editors: R. Bhargava, S. Fujii, T. J. Kröncke, and L. Lampmann

Copyright (C) 2012 Mohammed Mebarki et al. This is an open access article distributed under the Creative Commons Attribution License, which permits unrestricted use, distribution, and reproduction in any medium, provided the original work is properly cited.

\begin{abstract}
Ovarian cystadenofibroma is a relatively rare tumor; it is usually asymptomatic and is found incidentally. We present the case of a 24-year-old female patient, who had undergone total thyroidectomy for thyroid papillary carcinoma, with an asymptomatic giant cystadenofibroma, incidentally discovered by diagnostic ${ }^{131} \mathrm{I}$ ISPECT/CT WBSs. We summarize the clinical history, imaging data, and histopathological study on a rare case of radioiodine accumulation in cystadenofibroma, and we discuss the mechanism of uptake of radioiodine in this case.
\end{abstract}

\section{Introduction}

Whole-body scans (WBSs) after administration of diagnostic or therapeutic doses of ${ }^{131}$ I have a high sensitivity in detecting physiological and/or pathological uptake of radioiodine [1]. However, several unusual lesions can cause false-positive results $[2,3]$. One of the main limitations of two dimensional planar ${ }^{131}$ I-WBSs imaging is its lack of anatomical details. Single photon emission computed tomography/computed tomography (SPECT/CT) can correctly localize and characterize lesions presenting radioiodine uptake [4].

We present a rare case, an incidental finding of cystadenofibroma with radioiodine accumulation on follow-up diagnostic ${ }^{131} \mathrm{I}-\mathrm{WBS}$ s with SPECT/CT.

\section{Case Presentation}

A 24-year-old female patient underwent total thyroidectomy with central and bilateral lymphadenectomy at the age of 21 years, histopathology revealed papillary thyroid carcinoma and positive lymph nodes.
She was treated with $5.55 \mathrm{GBq}$ radioiodine $\left({ }^{131} \mathrm{I}\right)$ when the serum thyroid-stimulating hormone level was $100 \mu \mathrm{IU} / \mathrm{mL}$, and the serum thyroglobulin concentration was $68.7 \mathrm{ng} / \mathrm{mL}$.

Follow-up diagnostic ${ }^{131}$ I-WBS, 72 hours after oral administration of $370 \mathrm{MBq}$ of ${ }^{131} \mathrm{I}$, performed on a gamma camera equipped with a high-energy all-purpose parallelhole collimator (GE Infinia Hawkeye 4 slices), revealed a large rounded focus of radioiodine accumulation in the lower abdomen and pelvis. No abnormal radioiodine uptake was seen in the neck region (Figure 1).

Low-dose-integrated SPECT-CT of the abdomen and pelvis was then performed on the same gamma camera demonstrating a very large abdominal mass in the transverse coronal and sagittal images with heterogeneous distribution of ${ }^{131}$ I throughout the tumour mass (Figure 2).

At the time of diagnostic ${ }^{131}$ I-WBS the serum TSH level was $100 \mu \mathrm{IU} / \mathrm{mL}$ and the serum thyroglobulin concentration was $20 \mathrm{ng} / \mathrm{mL}$.

Gynaecological anamnesis reported menarche appeared at the age of 14, regular menstrual cycles, a two-month 


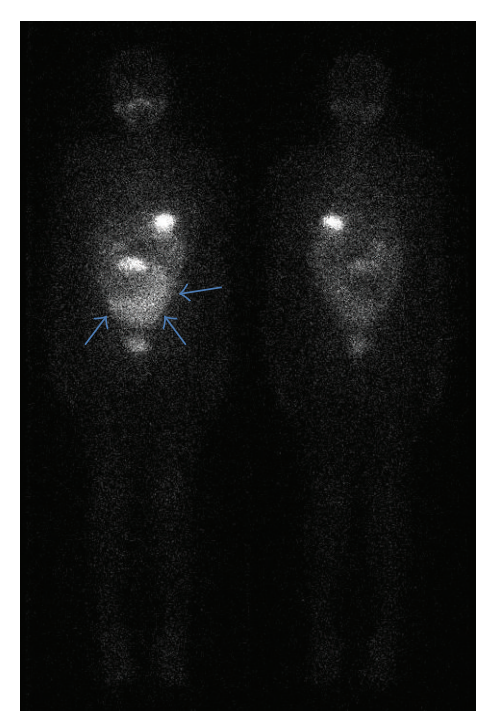

Figure 1: A 24-year-old female patient who had undergone total thyroidectomy with central and bilateral neck dissection for papillary thyroid cancer treated with radioiodine after. Anterior and posterior whole-body planar imaging (left and right) obtained 72 hours after oral administration of a diagnostic dose of $740 \mathrm{MBq}$ of ${ }^{131}$ I. They show a large rounded focus of activity in the lower abdomen and pelvis. There is no abnormal radioiodine uptake in the neck areas.
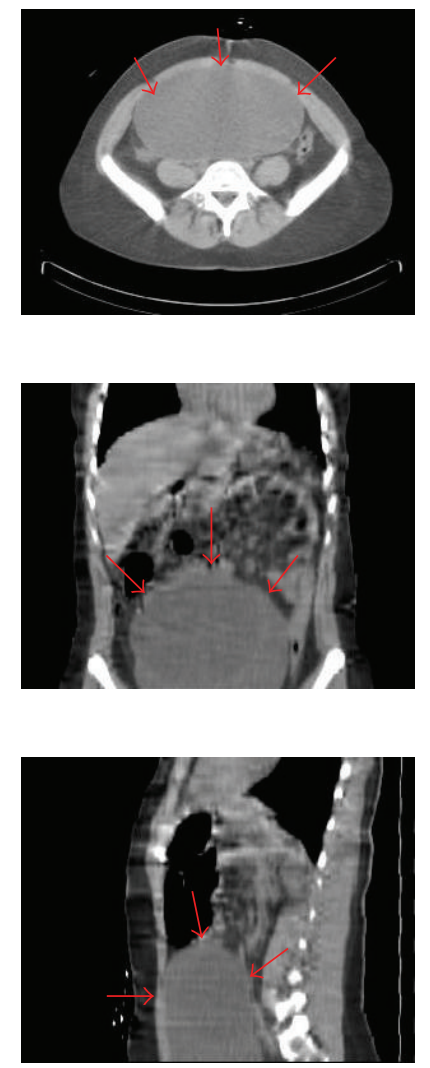

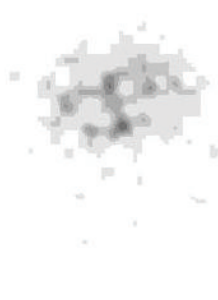

(a)

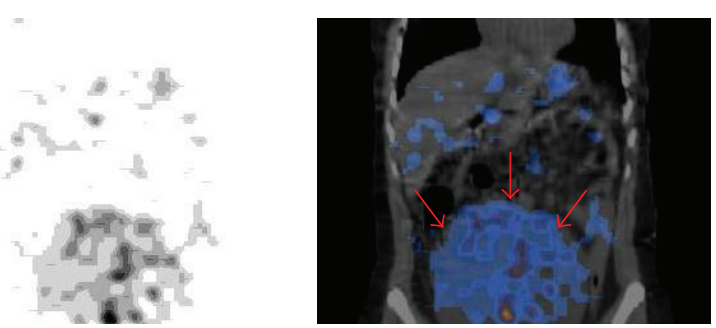

(b)
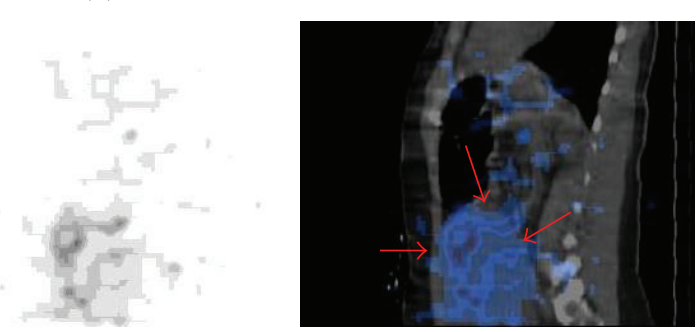

(c)

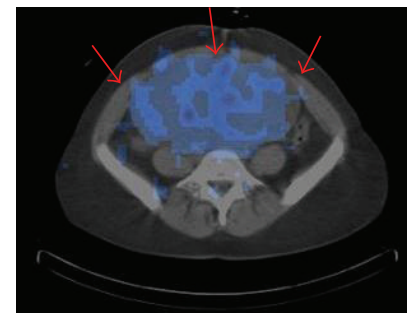

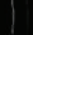

FIgure 2: Abdominal SPECT-CT image (left, CT; middle, SPECT; right, ${ }^{131}$ I-SPECT/CT) revealed a very large abdominal mass with radioiodine accumulation in the transaxial (a) coronal (b), and sagittal (c) images. Note the heterogeneous distribution of radioiodine throughout the tumour mass. 


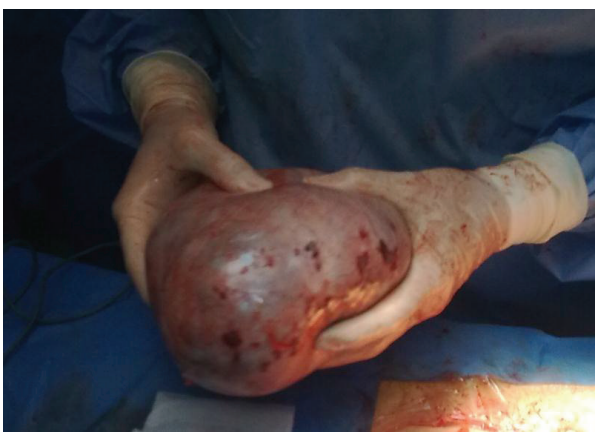

Figure 3: Gross photograph surgical specimen shows tumor, measuring $19 \times 17 \times 16 \mathrm{~cm}$ in the left ovary.

history of swelling in the lower abdomen, gradual abdominal enlargement, discomfort, and urinary compressive symptoms; these clinical manifestations were neglected by the patient.

One month later, laparotomy was performed and the ovarian mass, measuring approximately $19 \times 17 \times 16 \mathrm{~cm}$, was completely resected (Figure 3 ). Histopathological analysis revealed a borderline serous ovarian cystadenofibroma, the existence of thyroid tissue was excluded. The patient is well on followup.

\section{Discussion}

This common benign ovarian tumor is frequently incidentally discovered in patients without clinical evidence. We describe an unusual case of incidental detection of such tumors in a ${ }^{131} \mathrm{I}-\mathrm{WBS}$ with SPECT-CT.

Previous incidental findings of benign and malignant ovarian tumors on planar whole body scan have been described in the literature [5-9]. To our knowledge, only two previous cases of radioiodine uptake in a cystadenofibroma have been reported until March 2012. Flug et al. have reported a case of radioiodine accumulation in a large adnexal cystadenofibroma [10]. Song et al. have reported a case of false-positive ${ }^{131} \mathrm{I}$ uptake by an ovarian serous cystadenofibroma [11].

The mechanism of radioiodine accumulation in this ovarian tumor is still poorly understood. We propose two possible theories, one based on the nonspecific radioiodine accumulation in inflamed tissues, the other based on the active transport of radioiodine due to the presence of the sodium iodide symporter (NIS) in the tumor cells [10, 11].

In conclusion, a wider use of SPECT/CT in the followup of patients with differentiated thyroid carcinoma is suggested, particularly when planar ${ }^{131} \mathrm{I}-\mathrm{WBS}$ s is not conclusive.

The presence of abnormal pelvic uptake of radioiodine in WBSs needs a gynecological examination and radiological exploration $[12,13]$.

Patient clinical information and prior radiological investigations are crucial elements for WBSs interpretation.

\section{References}

[1] M. R. Carlisle, C. Lu, and I. R. McDougall, "The interpretation of ${ }^{131} \mathrm{I}$ scans in the evaluation of thyroid cancer, with an emphasis on false positive findings," Nuclear Medicine Communications, vol. 24, no. 6, pp. 715-735, 2003.

[2] A. Spanu, M. E. Solinas, F. Chessa, D. Sanna, S. Nuvoli, and G. Madeddu, "131 I SPECT/CT in the follow-up of differentiated thyroid carcinoma: incremental value versus planar imaging," Journal of Nuclear Medicine, vol. 50, no. 2, pp. 184-190, 2009.

[3] O. Turker, I. Dogan, and K. Kumanlioglu, "Radioiodine accumulation in a large adnexal cystadenofibroma," Thyroid, vol. 20, no. 5, pp. 561-562, 2010.

[4] H. Wakabayashi, K. Nakajima, M. Fukuoka et al., "Doublephase ${ }^{131}$ I whole body scan and 131I SPECT-CT images in patients with differentiated thyroid cancer: their effectiveness for accurate identification," Annals of Nuclear Medicine, vol. 25, no. 9, pp. 609-615, 2011.

[5] O. Morel, V. Rohmer, S. Girault, J. P. Muratet, C. Berthelot, and P. Jallet, "Abnormal iodine-131 uptake in a benign mucinous ovarian cystadenoma mimicking struma ovarii," Clinical Nuclear Medicine, vol. 32, no. 1, pp. 64-66, 2007.

[6] Z. L. Qiu, Y. H. Xu, H. J. Song, and Q. Y. Luo, "Unusual ${ }^{131}$ I uptake in a benign mucinous cystadenoma of the ovary in a patient with papillary thyroid cancer," Clinical Nuclear Medicine, vol. 35, no. 12, pp. 965-966, 2010.

[7] S. Srivatsa and R. K. Halkar, "I-131 Uptake in an endometriotic ovarian cyst in a patient with papillary thyroid carcinoma," Endocrinologist, vol. 20, no. 5, pp. 228-229, 2010.

[8] S. T. Lim, H. J. Jeong, M. J. Chung, C. Y. Yim, and M. H. Sohn, "Malignant struma ovarii demonstrated on post-therapy radioiodine scan after total thyroidectomy for papillary thyroid cancer," Clinical Nuclear Medicine, vol. 33, no. 6, pp. 429-431, 2008.

[9] D. Utsunomiya, S. Shiraishi, K. Kawanaka et al., "Struma ovarii coexisting with mucinous cystadenoma detected by radioactive iodine," Clinical Nuclear Medicine, vol. 28, no. 9, pp. 725-727, 2003.

[10] J. Flug, K. Lameka, R. Lee, D. S. Katz, W. W. Sung, and E. Yung, "False-positive I-131 uptake by an ovarian serous cystadenofibroma," Clinical Nuclear Medicine, vol. 37, pp. 178-180, 2012.

[11] H. J. Song, Y. L. Xue, Y. H. Xu, Z. L. Qiu, and Q. Y. Luo, "Abnormal ${ }^{131} \mathrm{I}$ uptake in a benign serous ovarian cystadenoma mimicking bladder physiological uptake," Clinical Nuclear Medicine, vol. 37, pp. 59-60, 2012.

[12] O. Kraft, L. Mrhac, P. Sirucek et al., "I-131 uptake in an ovarian tumor: differential diagnosis for abdominal I-131 uptake," Clinical Nuclear Medicine, vol. 34, no. 6, pp. 390-392, 2009.

[13] I. Rachinsky and A. Driedger, "Iodine-131 uptake in a menstruating uterus: value of SPECT/CT in distinguishing benign and metastatic iodine-positive lesions," Thyroid, vol. 17, no. 9, pp. 901-902, 2007.

\section{Conflict of Interests}

The authors declare that they have no conflict of interests. 


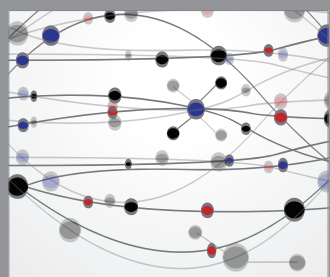

The Scientific World Journal
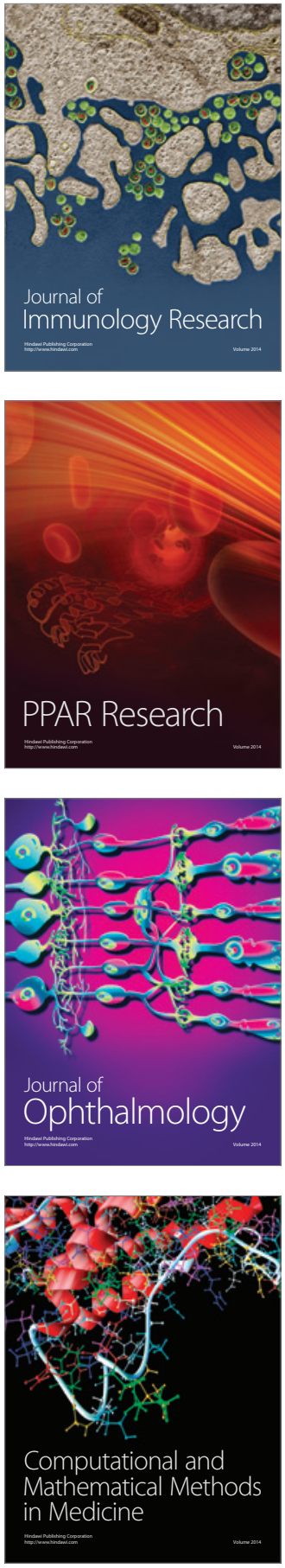

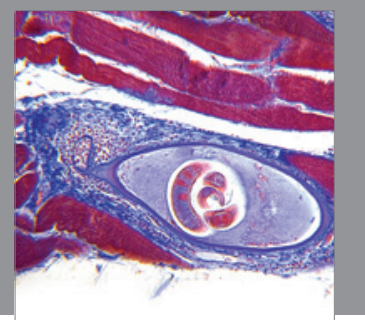

Gastroenterology

Research and Practice
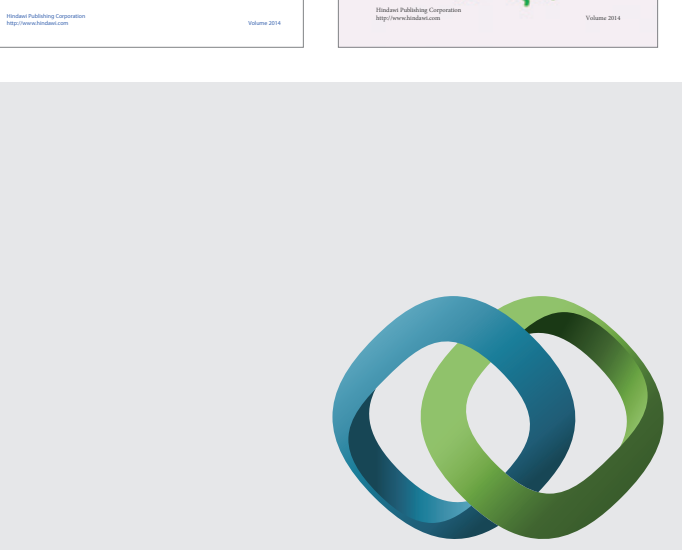

\section{Hindawi}

Submit your manuscripts at

http://www.hindawi.com
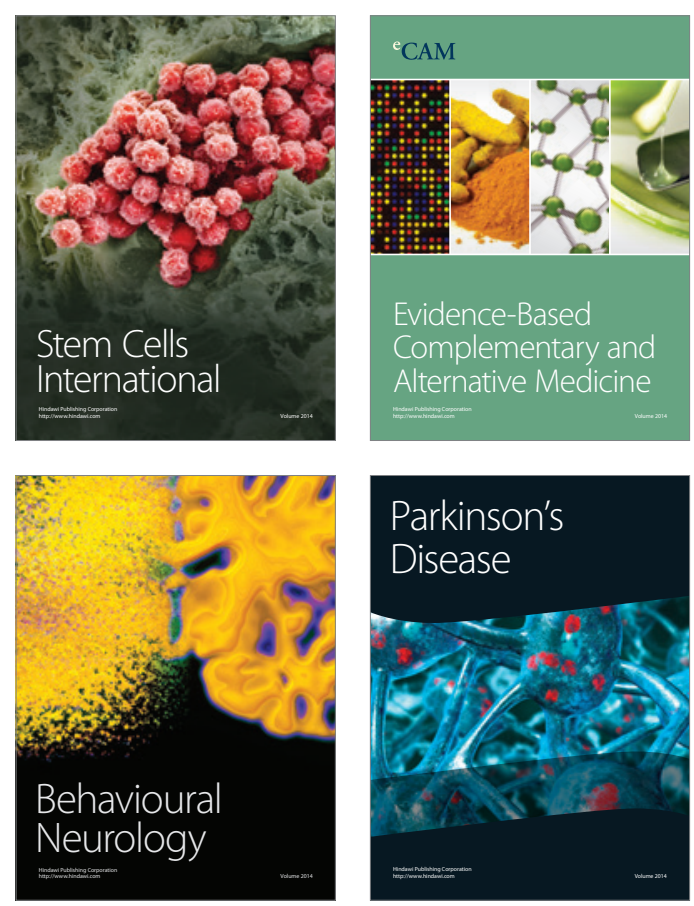

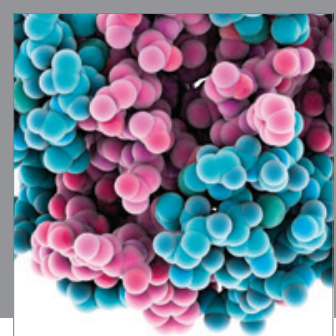

Journal of
Diabetes Research

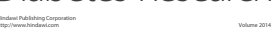

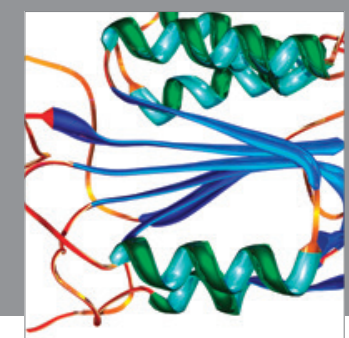

Disease Markers
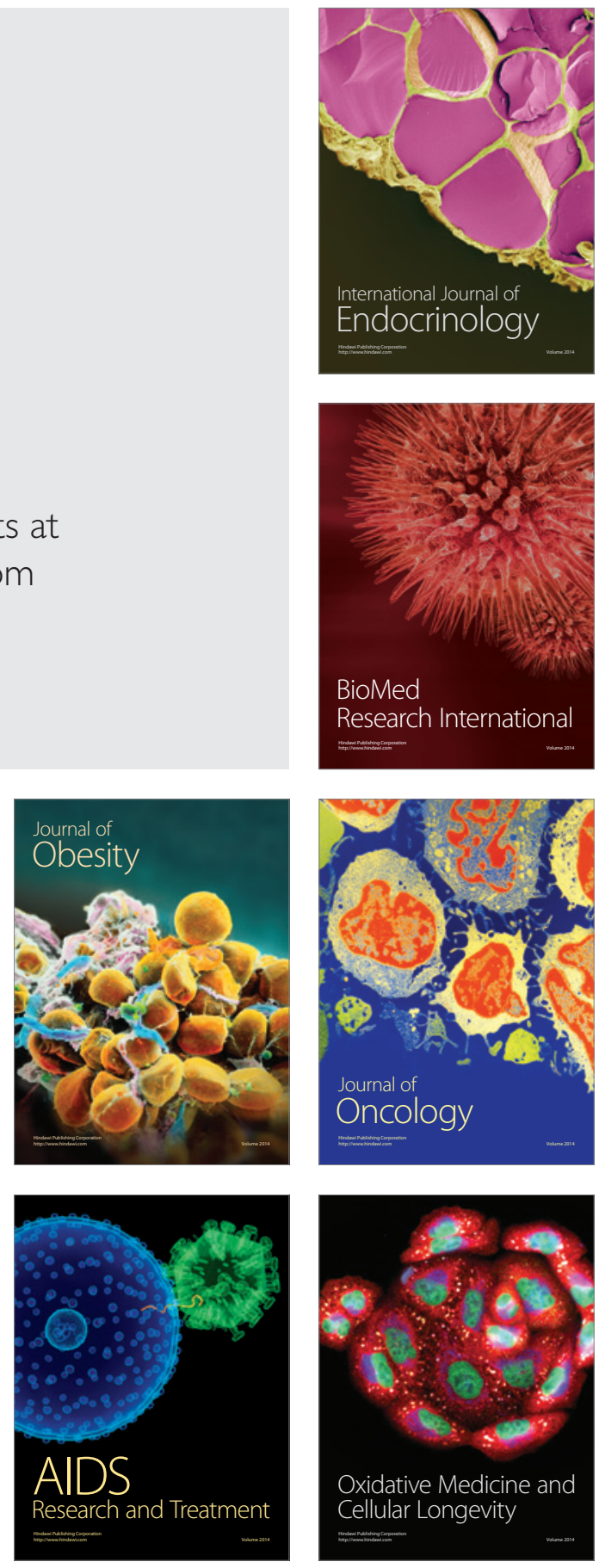